\title{
The impact of remission and coexisting migraine on anxiety and depression in cluster headache
}

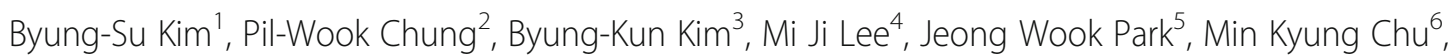
Jin-Young Ahn ${ }^{7}$, Dae Woong Bae ${ }^{8}$, Tae-Jin Song ${ }^{9}$, Jong-Hee Sohn ${ }^{10}$, Kyungmi Oh ${ }^{11}$, Daeyoung Kim ${ }^{12}$, Jae-Moon Kim ${ }^{12}$, Soo-Kyoung Kim ${ }^{13}$, Yun-Ju Choi ${ }^{14}$, Jae Myun Chung ${ }^{15}$, Heui-Soo Moon ${ }^{2}$, Chin-Sang Chung ${ }^{4}$, Kwang-Yeol Park ${ }^{16^{*}+}$ and Soo-Jin Cho ${ }^{17^{*+}}$ (D)

\begin{abstract}
Background: Our aim was to investigate the relationship between coexisting cluster headache $(\mathrm{CH})$ and migraine with anxiety and depression during active cluster bouts, and how symptoms change during remission.

Methods: We analyzed data from 222 consecutive $\mathrm{CH}$ patients and 99 age- and sex-matched controls using a prospective multicenter registry. Anxiety or depression was evaluated using the Generalized Anxiety Disorder-7 (GAD-7) or Patient Health Questionnaire-9 (PHQ-9), respectively. Moderate-to-severe anxiety or depression was defined as a score of $\geq 10$ at baseline (during a cluster bout). We assessed for changes in anxiety and depression during $\mathrm{CH}$ remission periods.
\end{abstract}

Results: Among the $\mathrm{CH}$ patients, the prevalence of moderate-to-severe anxiety and depression was seen in 38.2\% and $34.6 \%$, respectively. Compared with controls, $\mathrm{CH}$ patients were associated with moderate-to-severe anxiety and depression (multivariable-adjusted odds ratio $[\mathrm{aOR}]=7.32,95 \%$ confidence intervals $[\mathrm{Cl}]=3.35-15.99$ and $\mathrm{aOR}=4.95$, $95 \% \mathrm{Cl}=2.32-10.57$, respectively). CH patients with migraine were significantly more likely to have moderate-tosevere anxiety and depression $(\mathrm{aOR}=32.53,95 \% \mathrm{Cl}=6.63-159.64$ and $\mathrm{aOR}=16.88,95 \% \mathrm{Cl}=4.16-68.38$, respectively), compared to controls without migraine. The GAD-7 and PHQ-9 scores were significantly reduced between cluster bout and remission periods (from $6.8 \pm 5.6$ to $1.6 \pm 2.8 ; P<0.001$, and from $6.1 \pm 5.0$ to $1.8 \pm 2.4 ; P<$ 0.001 , respectively).

Conclusions: Our results indicate that $\mathrm{CH}$ patients are at increased risk of anxiety and depression, especially in the presence of coexisting migraine. However, the anxiety and depression can improve during remission periods.

Keywords: Anxiety, Cluster headache, Depression, Headache, Migraine

\footnotetext{
*Correspondence: kwangyeol.park@gmail.com; dowonc@naver.com

${ }^{\dagger}$ Kwang-Yeol Park and Soo-Jin Cho contributed equally to this work.

${ }^{16}$ Department of Neurology, Chung-Ang University Hospital, 102

Heukseok-ro, Dongjak-gu, Seoul 06973, South Korea

${ }^{17}$ Department of Neurology, Dongtan Sacred Heart Hospital, Hallym

University College of Medicine, Keun Jae Bong-gil 7, Hwaseong, Gyeonggi-do 18450, South Korea

Full list of author information is available at the end of the article
}

(C) The Author(s). 2020 Open Access This article is licensed under a Creative Commons Attribution 4.0 International License, which permits use, sharing, adaptation, distribution and reproduction in any medium or format, as long as you give appropriate credit to the original author(s) and the source, provide a link to the Creative Commons licence, and indicate if changes were made. The images or other third party material in this article are included in the article's Creative Commons. licence, unless indicated otherwise in a credit line to the material. If material is not included in the article's Creative Commons licence and your intended use is not permitted by statutory regulation or exceeds the permitted use, you will need to obtain permission directly from the copyright holder. To view a copy of this licence, visit http://creativecommons.org/licenses/by/4.0/. The Creative Commons Public Domain Dedication waiver (http://creativecommons.org/publicdomain/zero/1.0/) applies to the data made available in this article, unless otherwise stated in a credit line to the data. 


\section{Introduction}

Cluster headache $(\mathrm{CH})$ is a rare primary headache disorder characterized by recurrent excruciatingly severe unilateral pain in association with ipsilateral cranial autonomic features [1-3]. The name cluster headache stems from the tendency of the pain attacks to cluster together within bouts that usually last several weeks, which is unlike migraine and any other primary headache disorder. Approximately $80-90 \%$ of $\mathrm{CH}$ patients have episodic cluster headaches $(\mathrm{ECH})$, where active cluster bouts are separated by remissions lasting more than 3 months.

In primary headaches, mainly migraine, psychiatric comorbidities are important because they can potentially impact the clinical course, quality of life, and headache management negatively [4-8]. Previous studies have shown that $\mathrm{CH}$ patients can have psychiatric comorbidities such as anxiety and depression [9-16]. In addition, depressive symptoms can influence disease burden [14]. However, whether the risk of anxiety and depression differs according to the status of the cluster headaches (active bout vs. remission) has not been investigated in detail to date $[11,17,18]$. In addition, in a subset of $\mathrm{CH}$ patients ranging from $10.0 \%$ to $16.7 \%$, migraine has been reported to coexist [19-22]. Given the well-recognized link between migraine and psychiatric comorbidities, coexisting migraine may independently influence the risk for anxiety and depression in $\mathrm{CH}$ patients, although this is a relatively unexplored area to date.

Considering the debilitating nature of repetitive $\mathrm{CH}$ attacks during an active cluster bout, we hypothesized that $\mathrm{CH}$ patients were at an increased risk for anxiety and depression in active bout period, but that their anxiety and depression would reduce during remission. In addition, given the relationship between migraine and psychiatric comorbidities, we further hypothesized that the risk for anxiety and depression could be influenced by coexisting migraine in $\mathrm{CH}$ patients. To test these hypotheses, we conducted a prospective study based on data from a multicenter $\mathrm{CH}$ registry to investigate the associations between $\mathrm{CH}$, coexisting migraine, anxiety, and depression during an active episode of cluster bout (acting as the baseline period) and changes in anxiety and depression during remission periods.

\section{Methods}

\section{Participants and study design}

This cross-sectional study was planned as a part of the Korean Cluster Headache Registry (KCHR) study, a prospective, multicenter registry enrolling consecutive $\mathrm{CH}$ patients aged $\geq 19$ years at 16 hospitals across Korea - 14 university hospitals (eight tertiary and six secondary referral centers) and 2 secondary referral general hospitals. Patient enrollment was conducted between September
2016 and December 2018 following Institutional Review Board (IRB) approval in each hospital. For the present study, among all the $\mathrm{KCHR}$ participants, we enrolled only those with $\mathrm{CH}$ compatible with the third edition of The International Classification of Headache Disorders (ICHD-3) [23]. Comorbid migraine was also determined based on the ICHD-3. The detailed protocol of the KCHR has been published elsewhere previously [24-26]. The study protocol was approved by the IRB in each study hospital and complied with the Declaration of Helsinki and Good Clinical Practice guidelines. All patients fully understood the study aims and gave informed written consent before their participation. Due to the nature of our research question, we included patients who enrolled during the cluster bout period of $\mathrm{CH}$.

Control subjects without $\mathrm{CH}$ were recruited from 3 out of the 16 hospitals, and were matched on the basis of age and sex. Volunteers aged 19-65 years were invited to participate in this study as a control group, as long as they had no history of diabetes, thyroid illness, severe obesity, severe hepatic or renal illness, or malignancy, and they had the cognitive capacity to complete the questionnaires. Many of the control group participants were friends or relatives of patients with headaches, or employees of the hospital. We evaluated all of the control group participants to determine the presence of migraine based on the ICHD-3. Eligibility of the rest of controls was being headache free $(<1$ headache day per month) with no previous history of primary or secondary headache disorder. All control subjects were enrolled after receiving informed written consent.

\section{Measurement}

The KCHR included demographic and clinical data that were collected during the last cluster bout. Anxiety and depression were assessed using the Korean versions of the Generalized Anxiety Disorder 7-item scale (GAD-7) and the Patient Health Questionnaire 9-item scale (PHQ-9). Each item of GAD-7 and PHQ-9 was measured on a four-point scale, from 0 to $3(0=$ never, $1=$ several days, $2=$ more than half the time, and $3=$ nearly every day). Items were scored based on occurrence over the previous 2 weeks. The total scores of GAD-7 and PHQ-9 ranged from 0 to 21 and from 0 to 27, respectively. Based these scores, the patients were classified into three anxiety and depression severity groups: no anxiety (GAD-7 score: 0-4), mild anxiety (GAD-7 score: $5-9$ ), and moderate-to-severe anxiety (GAD-7 score: 10-21), and no depression (PHQ-9 score: 0-4), mild depression (PHQ-9 score: 5-9), and moderate-to-severe depression (PHQ-9 score: 10-27) [18].

Regarding the presence of anxiety, study participants were divided dichotomously into the groups of no anxiety (GAD-7 score $\leq 4)$ or any anxiety (GAD-7 score $\geq 5$ ) 
group. The same was done for depression, with the two groups being a no depression group (PHQ-9 score $\leq 4$ ) and an any depression (PHQ-9 score $\geq 5$ ) group. During follow-up, $\mathrm{CH}$ patients were asked to repeat the tests of GAD-7 and PHQ-9 when their cluster bout status subsided into remission. Given that GAD-7 and PHQ-9 evaluate the status of anxiety and depression within the last 2 weeks, $\mathrm{CH}$ patients performed the follow-up tests of GAD-7 and PHQ-9 after 2 weeks following the end of their cluster bout. During the remission period, we evaluated for a change in GAD-7 and PHQ-9 scores between the cluster bout and the remission period. The exclusion criteria for the remission period analysis were as follows: [1] chronic cluster headache $(\mathrm{CCH})$ [2]; no, or incomplete, response to the GAD-7 or PHQ-9 questionnaire [3]; follow-up GAD-7 and PHQ-9 testing performed within 2 weeks of the end of their cluster bout; and [4] uncertainty as to the end date of the active cluster bout.

\section{Statistical analysis}

To test the study hypothesis, $\mathrm{CH}$ patients and control subjects were classified into 4 groups according to the presence of coexisting migraine: a $\mathrm{CH}$ with migraine group, a $\mathrm{CH}$ without migraine group, a control group with migraine, and a control group without migraine. Continuous variables are presented as mean values \pm standard deviation, and categorical variables are presented as numbers (percentage). The Chi-square test for categorical variables and the one-way ANOVA test for continuous variables were used for the assessment of statistical significance of intergroup differences. To identify whether $\mathrm{CH}$ and coexisting migraine were associated with anxiety and depression in $\mathrm{CH}$, we performed a logistic regression analysis. The results of the univariate analyses are presented as odds ratios (ORs) accompanied by $95 \%$ confidence intervals (CIs). To determine whether $\mathrm{CH}$ and coexisting migraine were independent variables contributing to anxiety and depression in $\mathrm{CH}$, we calculated multivariable-adjusted odds ratios (aORs) and 95\% CIs after adjusting for potential covariates. To assess for a change in anxiety and depression scores between active cluster bout and remission periods (remission period analysis), we used the paired t-test for the continuous data and the McNemar test for the dichotomous groups we created, as mentioned above. Since we expected a reduction in the GAD-7 and PHQ-9 scores during remission, in those with GAD-7 and/or PHQ-9 scores $\geq 5$ during an acute cluster bout we compared the proportion of participants experiencing a more than $50 \%$ reduction in the GAD-7 and PHQ-9 scores, according to prespecified subgroups (age $<40$ years vs. age $\geq 40$ years, male sex vs. female sex, and presence of migraine vs. lack of migraine). All statistical analyses were performed using SPSS for Windows Version 18.0 (SPSS Inc.,
Chicago, IL, USA). All $P$-values reported as $<0.05$ in two-tailed tests were considered statistically significant.

\section{Results \\ Study participants, anxiety, and depression}

During the study period, $222 \mathrm{CH}$ patients and 99 ageand sex-matched controls were enrolled in the multicenter headache registry (Fig. 1). Baseline characteristics between the $\mathrm{CH}$ patients and controls are shown in Table 1. No significant differences were observed across the groups of the $\mathrm{CH}$ patients and controls in terms of age, sex, current smoking status, and alcohol consumption status. With respect to the $\mathrm{CH}$ subtype, of the 222 $\mathrm{CH}$ patients, 157 (70.7\%), 11 (4.9\%), and 24 (10.8\%) patients were classified as having $\mathrm{ECH}$ (code 3.1.1), $\mathrm{CCH}$ (code 3.1.2), and probable $\mathrm{CH}$ (code 3.5.1), respectively. For the remaining 33 patients (14.8\%), their first episode of $\mathrm{CH}$ did not turn into remission within 1 year of their onset or did not follow more than 1 year, so they were classified neither $\mathrm{ECH}$ nor $\mathrm{CCH}$, their diagnosis was finally coded as 3.1.

Among the $\mathrm{CH}$ patients, the prevalence of anxiety and depression was $67.5 \%$ and $59.5 \%$, respectively. The proportion of $\mathrm{CH}$ patients with moderate-to-severe anxiety was more than three times that seen in controls $(38.2 \%$ vs. $10.1 \%$ ), and the proportion of $\mathrm{CH}$ patients with depression was more than double that of controls (34.6\% vs. $12.1 \%)$. The prevalence of moderate-to-severe anxiety and depression was highest in the $\mathrm{CH}$ with migraine group $(51.6 \%$ and $45.2 \%$, respectively) relative to the $\mathrm{CH}$ without migraine group and the two control groups (with and without migraine). Regarding item of suicidal idea in PHQ-9, the $\mathrm{CH}$ patients reported any suicidal idea ( $\geq$ Several days, item score $\geq 1$ ) more frequently, in comparison with the controls $(26.1 \%$ vs. $10.1 \% ; P<0.001)$.

\section{Association of cluster headache and coexisting migraine with anxiety}

In univariable analyses, the $\mathrm{CH}$ patients were significantly more likely to experience any anxiety and moderate-to-severe anxiety compared to controls (Table 2). Those with coexisting $\mathrm{CH}$ and migraine had the highest $\mathrm{OR}$ for moderate-to-severe anxiety $(\mathrm{OR}=32.53$, 95\% CI = 6.73-157.12).

In multivariable analyses, the association between the presence of $\mathrm{CH}$, any anxiety, and moderate-to-severe anxiety remained significant after adjustment for potential covariates. Furthermore, the association between the control with migraine group, the $\mathrm{CH}$ without migraine group, and the $\mathrm{CH}$ with migraine group and both any anxiety and moderate-to-severe anxiety persisted after multivariable adjustment. The $\mathrm{CH}$ with migraine group was associated with an extremely high likelihood of moderate-to-severe anxiety $(\mathrm{aOR}=32.53,95 \% \mathrm{CI}=$ 6.63-159.64). 


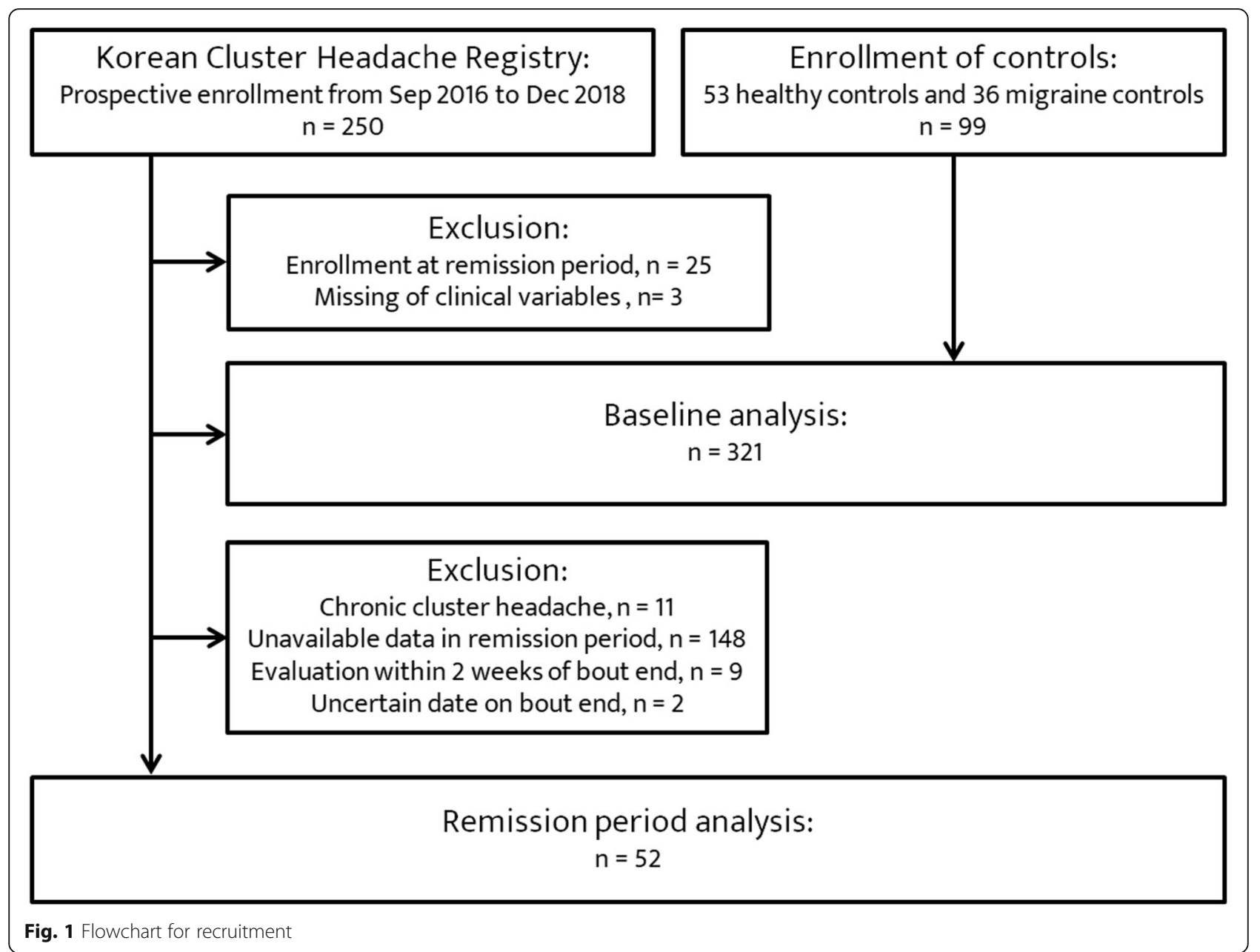

Table 1 Baseline characteristics between cluster headache patients and controls

\begin{tabular}{|c|c|c|c|c|c|}
\hline & \multicolumn{2}{|l|}{ Control } & \multicolumn{2}{|l|}{$\mathrm{CH}$} & \\
\hline & Without migraine & With migraine & Without migraine & With migraine & \\
\hline Variable & $(n=63)$ & $(n=36)$ & $(n=191)$ & $(n=31)$ & $P$ \\
\hline Age, years & $37.6 \pm 10.2$ & $34.8 \pm 7.4$ & $38.3 \pm 11.1$ & $37.2 \pm 8.1$ & 0.312 \\
\hline Female sex, n (\%) & $11(17.5)$ & $6(16.7)$ & $26(13.6)$ & $9(29.0)$ & 0.188 \\
\hline Current smoking, n (\%) & $20(31.7)$ & $13(36.1)$ & $89(46.6)$ & $7(22.6)$ & 0.025 \\
\hline Alcohol drinking, n (\%) & $42(66.7)$ & $15(41.7)$ & $98(51.3)$ & $10(32.3)$ & 0.008 \\
\hline GAD-7 score & $2.7 \pm 3.0$ & $6.2 \pm 4.6$ & $7.7 \pm 5.5$ & $9.7 \pm 6.9$ & $<0.001$ \\
\hline Anxiety, n (\%) & & & & & $<0.001$ \\
\hline None/minimal (GAD-7: 0-4) & $50(79.4)$ & $15(41.7)$ & $63(33.0)$ & $9(29.0)$ & \\
\hline Mild (GAD-7: 5-9) & $11(17.5)$ & $13(36.1)$ & $59(30.9)$ & $6(19.4)$ & \\
\hline Moderate-to-severe (GAD-7 $\geq 10)$ & $2(3.2)$ & $8(22.2)$ & $69(36.1)$ & $16(51.6)$ & \\
\hline PHQ-9 score & $3.1 \pm 3.1$ & $6.6 \pm 5.2$ & $7.4 \pm 6.1$ & $9.4 \pm 7.7$ & $<0.001$ \\
\hline Depression, n (\%) & & & & & $<0.001$ \\
\hline None/minimal (PHQ-9: 0-4) & $48(76.2)$ & $17(47.2)$ & $77(40.3)$ & $13(41.9)$ & \\
\hline Mild (PHQ-9: 5-9) & $12(19.0)$ & $10(27.8)$ & $51(26.7)$ & $4(12.9)$ & \\
\hline Moderate-to-severe (PHQ-9 $\geq 10$ ) & $3(4.8)$ & $9(25.0)$ & $63(33.0)$ & $14(45.2)$ & \\
\hline
\end{tabular}

The data are shown as the mean \pm standard deviation or number (percentage)

Abbreviations: CH Cluster headache; GAD-7 Generalized Anxiety Disorder (7-item scale); PHQ-9 Patient Health Questionnaire (9-item scale) 
Table 2 Association of cluster headache and coexisting migraine with anxiety

\begin{tabular}{|c|c|c|c|c|c|c|c|c|}
\hline & \multicolumn{4}{|c|}{ Any anxiety (GAD-7 scores $\geq 5$ ) } & \multicolumn{4}{|c|}{ Moderate-to-severe anxiety (GAD-7 scores $\geq 10$ ) } \\
\hline & Crude OR (95\% Cl) & $P$ & $\mathrm{aOR}(95 \% \mathrm{Cl})$ & P & Crude OR $(95 \% \mathrm{Cl})$ & $P$ & $\mathrm{aOR}(95 \% \mathrm{Cl})$ & P \\
\hline \multicolumn{9}{|l|}{ Presence of $\mathrm{CH}^{\mathrm{a}}$} \\
\hline Control & reference & & reference & & reference & & reference & \\
\hline $\mathrm{CH}$ & $3.98(2.41-6.57)$ & $<0.001$ & $5.17(2.93-9.12)$ & $<0.001$ & $5.52(2.72-11.20)$ & $<0.001$ & $7.32(3.35-15.99)$ & $<0.001$ \\
\hline \multicolumn{9}{|l|}{ Category of $\mathrm{CH}$ and migraine ${ }^{\mathrm{b}}$} \\
\hline Control without migraine & reference & & reference & & reference & & reference & \\
\hline Control with migraine & $5.38(2.18-13.25)$ & $<0.001$ & $5.29(2.10-13.29)$ & $<0.001$ & $8.71(1.73-43.71)$ & 0.009 & $8.43(1.65-42.96)$ & 0.01 \\
\hline $\mathrm{CH}$ without migraine & $7.81(3.95-15.43)$ & $<0.001$ & $7.71(3.84-15.49)$ & $<0.001$ & $17.25(4.09-72.73)$ & $<0.001$ & $16.01(3.77-68.03)$ & $<0.001$ \\
\hline $\mathrm{CH}$ with migraine & $9.40(3.50-25.22)$ & 0.001 & 9.78 (3.55-26.92) & 0.001 & $32.53(6.73-157.12)$ & $<0.001$ & $32.53(6.63-159.64)$ & $<0.001$ \\
\hline
\end{tabular}

${ }^{a}$ Adjustment for age, female sex, current smoking, alcohol drinking, and coexisting migraine

${ }^{\mathrm{b}}$ Adjustment for age, female sex, current smoking, and alcohol drinking

Abbreviations: $a O R$ Multivariable-adjusted odds ratio; $\mathrm{CH}$ Cluster headache; Cl Confidence interval; GAD-7 Generalized Anxiety Disorder (7-item scale); OR Odds ratio

\section{Association of cluster headache and coexisting migraine with depression}

In univariable analyses, the presence of $\mathrm{CH}$ was significantly associated with any depression and moderate-to-severe depression (Table 3). The $\mathrm{CH}$ with migraine group had the highest OR for the presence of moderate-to-severe depression $(\mathrm{OR}=16.47$, 95\% CI $=4.23-64.06$ ).

In multivariable analyses, the presence of $\mathrm{CH}$ had significant associations with any depression and moderate-to-severe depression after adjustment for potential covariates. Using the control without migraine group as a reference, the risk of moderate-tosevere depression was the highest in the group of patients with coexisting $\mathrm{CH}$ and migraine $(\mathrm{aOR}=$ 16.88, 95\% CI =4.16-68.38). Regarding $\mathrm{CH}$ and coexisting migraine, the $\mathrm{CH}$ patients with migraine had the biggest odds for suicidal idea of $\geq$ Several days (OR: 8.79, 95\% CI: 2.49-30.99; Supplementary Table).
Change of anxiety and depression between cluster bout and remission periods

Following the predefined exclusion criteria, data on 52 eligible $\mathrm{CH}$ patients were finally analyzed in the remission period analysis (Fig. 1). The 52 patients were followed for a median of 87.5 days (interquartile range: 42-131 days) between cluster bout and remission periods. The baseline characteristics of age, female sex, current smoking status, alcohol consumption status, coexisting migraine history, and the GAD-7 and PHQ-9 scores in this group did not significantly differ from those of the other 170 patients excluded from the remission period analysis.

The mean GAD-7 and PHQ-9 scores reduced significantly between the cluster bout and remission periods (from $6.8 \pm 5.6$ to $1.6 \pm 2.8 ; P<0.001$, and from $6.1 \pm 5.0$ to $1.8 \pm 2.4 ; P<0.001$, respectively; Fig. 2 ). During the remission period the prevalence of moderate-to-severe anxiety and depression was significantly decreased.

Table 3 Association of cluster headache and coexisting migraine with depression

\begin{tabular}{|c|c|c|c|c|c|c|c|c|}
\hline & \multicolumn{4}{|c|}{ Any depression (PHQ-9 scores $\geq 5$ ) } & \multicolumn{4}{|c|}{ Moderate-to-severe depression (PHQ-9 scores $\geq 10$ ) } \\
\hline & Crude OR (95\% Cl) & $P$ & $\mathrm{aOR}(95 \% \mathrm{Cl})$ & $P$ & Crude OR (95\% Cl) & $P$ & $\mathrm{aOR}(95 \% \mathrm{Cl})$ & $P$ \\
\hline \multicolumn{9}{|l|}{ Presence of $\mathrm{CH}^{\mathrm{a}}$} \\
\hline Control & reference & & reference & & reference & & reference & \\
\hline $\mathrm{CH}$ & $2.80(1.71-4.59)$ & $<0.001$ & $2.95(1.72-5.07)$ & $<0.001$ & $3.85(1.98-7.47)$ & $<0.001$ & $4.95(2.32-10.57)$ & $<0.001$ \\
\hline \multicolumn{9}{|l|}{ Category of $\mathrm{CH}$ and migraine ${ }^{b}$} \\
\hline Control without migraine & reference & & reference & & reference & & reference & \\
\hline Control with migraine & $3.57(1.49-8.57)$ & 0.004 & $3.24(1.31-8.05)$ & 0.011 & $6.66(1.67-26.58)$ & 0.007 & $6.07(1.45-25.31)$ & 0.013 \\
\hline $\mathrm{CH}$ without migraine & $4.73(2.47-9.05)$ & $<0.001$ & $4.20(2.16-8.18)$ & $<0.001$ & $9.84(2.97-32.62)$ & $<0.001$ & $8.63(2.55-29.18)$ & 0.001 \\
\hline $\mathrm{CH}$ with migraine & $4.43(1.76-11.11)$ & 0.002 & $4.07(1.58-10.52)$ & 0.004 & $16.47(4.23-64.06)$ & $<0.001$ & $16.88(4.16-68.38)$ & $<0.001$ \\
\hline
\end{tabular}

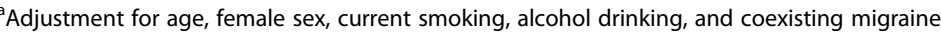

${ }^{\mathrm{b}}$ Adjustment for age, female sex, current smoking, and alcohol drinking

Abbreviations: aOR Multivariable-adjusted odds ratio; $\mathrm{CH}$ Cluster headache; $\mathrm{Cl}$ Confidence interval; OR Odds ratio; PHQ-9 Patient Health Questionnaire (9-item scale) 
A GAD-7 and PHQ-9 scores between cluster bout and remission
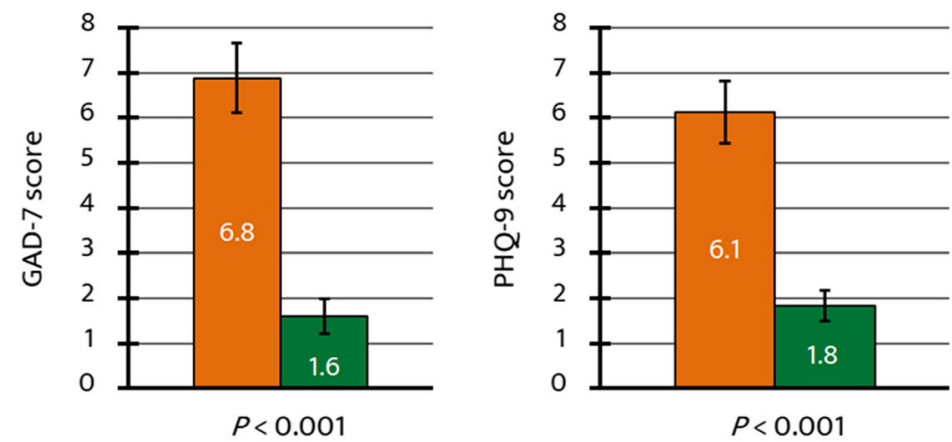

B Anxiety and depression between cluster bout and remission

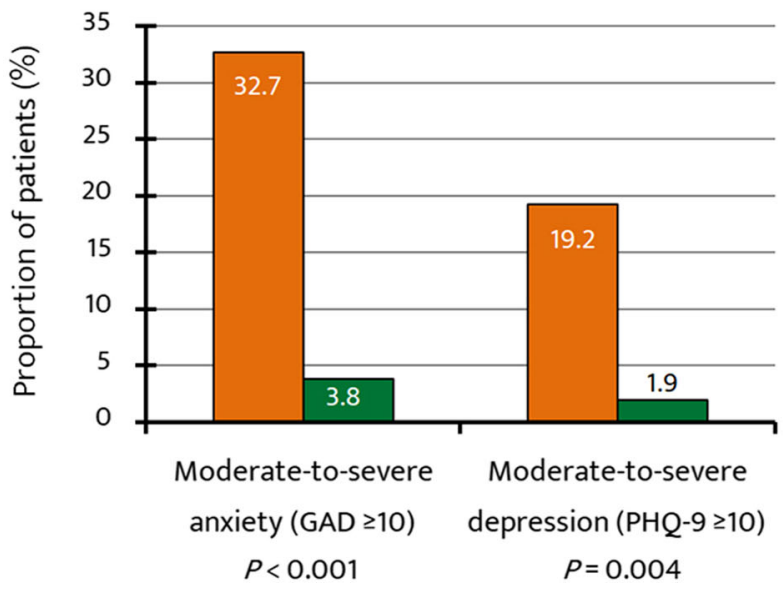

Fig. 2 a Changes in anxiety and depression scores between active cluster bouts and remission periods. $\mathbf{b}$ Changes in the proportion of patients with moderate-to-severe anxiety and depression between active cluster bouts and remission periods. Abbreviations: GAD-7, Generalized Anxiety Disorder (7-item scale); PHQ-9, Patient Health Questionnaire (9-item scale)

The proportions of patients with a more than $50 \%$ reduction in GAD-7 and PHQ-9 scores during the remission period were analyzed according to prespecified subgroups (Fig. 3). The proportion of patients with a more than 50\% reduction in GAD-7 and PHQ-9 scores was greater in patients without migraine than those with migraine (anxiety: $89.7 \%$ vs. $75.0 \%$, and depression: $85.2 \%$ vs. $50.0 \%$, respectively); however, the difference did not reach statistical significance.

\section{Discussion}

In the present study, we assessed for an association between $\mathrm{CH}$ and coexisting migraine with anxiety and depression, and for any changes between cluster bouts and remission periods. Compared to the control group, the $\mathrm{CH}$ patients were significantly more likely to have comorbid anxiety and depression after the multivariable adjustment including coexisting migraine. The associations differed according to the presence of coexisting migraine. Those in the $\mathrm{CH}$ with migraine group were at high risk of having moderate-to-severe anxiety and depression. In the remission period following a cluster bout the GAD-7 and PHQ-9 scores were significantly reduced, suggesting that anxiety and depression improve during the $\mathrm{CH}$ remission period.

Several early studies showed a wide range in the prevalence of psychiatric comorbidities in $\mathrm{CH}$. The estimated prevalence of anxiety and depression ranged from $11.8 \%$ to $75.7 \%$ and $6.3 \%$ to $43.0 \%$, respectively [9]. These results are insufficient to conclude that $\mathrm{CH}$ patients are at an increased risk for having psychiatric comorbidities. Meanwhile, a Taiwanese population study demonstrated that in $\mathrm{CH}$ patients without a history of psychiatric disorders, the risk for developing depression was 5.6 times higher than in control subjects [27]. Similarly, a largescale US study analyzing five-year insurance claims data demonstrated that $\mathrm{CH}$ patients, had 2.5 and 2.2 times higher odds of anxiety and depression compared to 


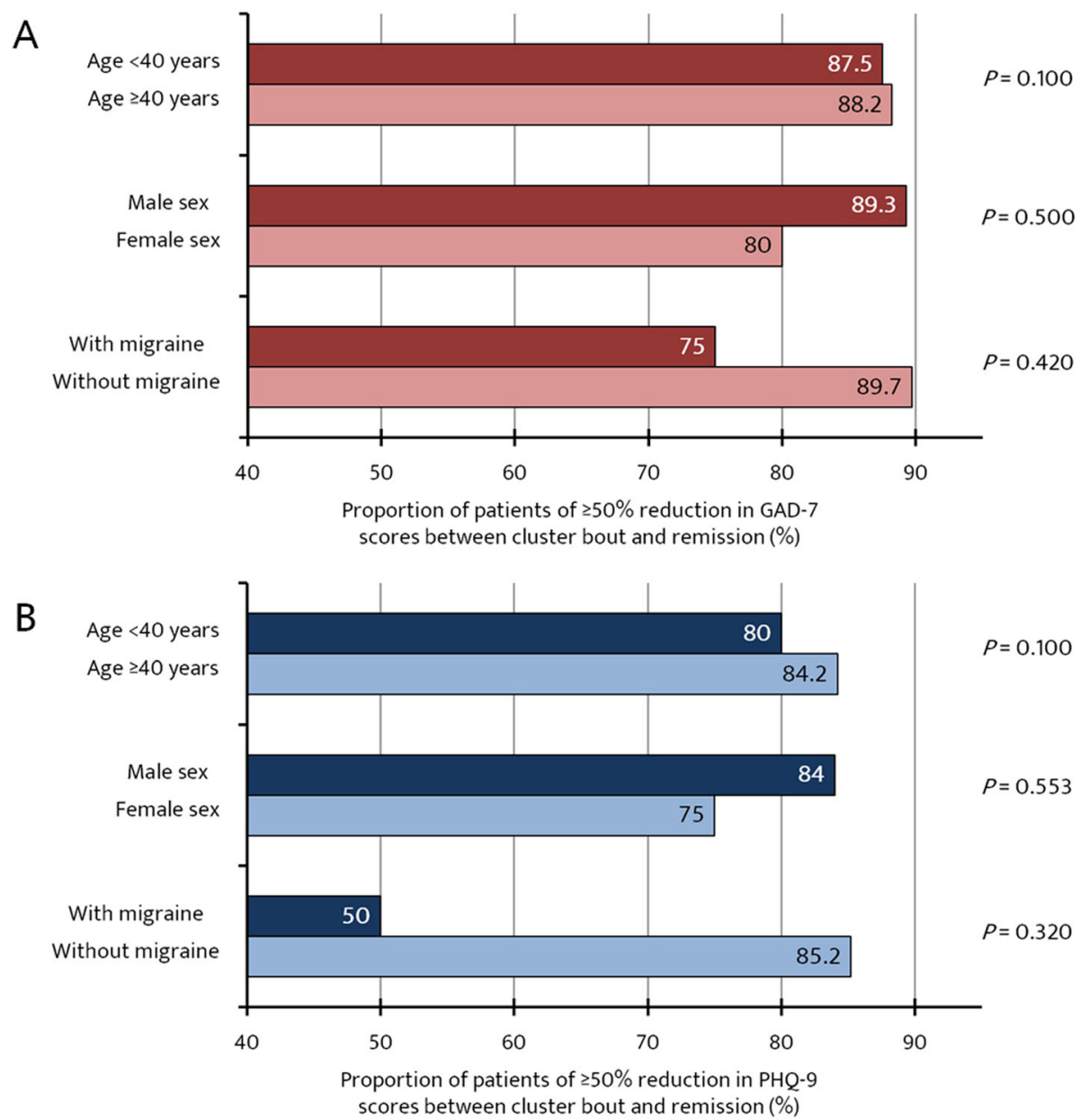

Fig. 3 Proportions of patients with a more than 50\% reduction in anxiety and depression according to prespecified subgroups. Abbreviations: GAD-7, Generalized Anxiety Disorder (7-item scale); PHQ-9, Patient Health Questionnaire (9-item scale)

controls, respectively [12]. In addition, a Dutch crosssectional study involving $462 \mathrm{CH}$ patients and 177 controls showed that $\mathrm{CH}$ patients had nearly double the anxiety and depression scores of control subjects [11]. In line with these findings, the current study can corroborate the notion that there is an increased risk of psychiatric comorbidities in $\mathrm{CH}$ patients.

In the present study, it is noteworthy that the $\mathrm{CH}$ with migraine group had approximately two-fold higher odds of moderate-to-severe anxiety and depression when compared to the $\mathrm{CH}$ without migraine group. This indicates that the bidirectional relationship between migraine and psychiatric comorbidities may further increase the risk of anxiety and depression among $\mathrm{CH}$ patients during a cluster bout. Given the debilitating nature of $\mathrm{CH}$, comorbid migraine often comes second place in terms of priority, and receives little or no attention in the clinical management of $\mathrm{CH}$ patients during the active cluster bout period. In this context, our findings imply that clinicians should not underestimate the presence and importance of coexisting migraine in the comprehensive and holistic management of $\mathrm{CH}$ that incorporates psychiatric well-being.

Despite the strong association between $\mathrm{CH}$, anxiety, and depression during active cluster bouts, anxiety and depression improved remarkably at remission in the present analysis. This suggests that the cyclical nature of $\mathrm{CH}$ also applies to the psychiatric comorbidities experienced in $\mathrm{CH}$ patients. Prior to our current research, few previous studies had examined this important issue. A German cross-sectional study showed that the proportion of patients with anxiety and depression was higher for patients with $\mathrm{ECH}$ at remission than those with $\mathrm{ECH}$ during an acute $\mathrm{CH}$ episode, which goes against our expectation [17]. A US pilot study conducted a cross-sectional comparison of the levels of anxiety and depression among ECH patients between active cluster bouts and remission periods [18]. The levels of anxiety and depression were similar irrespective of $\mathrm{CH}$ status. Like our 
study, a Dutch study found a strong association between active cluster bout and current depression [11]. However, the association was ultimately attenuated in the final model after adjusting for the scores of sleep disorders. Unlike those studies, our study has an advantage that the findings derive from a prospective observation of the temporal change of anxiety and depression.

We evaluated greater than $50 \%$ reductions in the anxiety and depression scale scores according to several prespecified subgroups. Our presumption was that anxiety and depression in female, older patients with coexisting migraine were less likely to improve during the remission period. We observed no significant difference in the subgroup analysis; however, it is interesting to note that the difference in the proportion of patients with more than a 50\% reduction in the anxiety and depression scales during remission was greatest between the subgroups of patients with coexisting migraine. This suggests that coexisting migraine has an important influence on the psychological burden of $\mathrm{CH}$ patients, and further studies of statistically sufficient sample size are needed to verify this.

Several hypotheses have been already proposed with regard to the risk of affective disorders in $\mathrm{CH}$ patients: 1) neuroimaging findings have shown shared anatomical substrates for the pain matrix and depression processing; 2) similarities between $\mathrm{CH}$ and depression have been observed in terms of hypothalamic dysfunction and chronobiology; and 3) common mood changes are reported in the pre- and post-ictal phases of a $\mathrm{CH}$ attack $[11,28-32]$. The possible mechanism underlying the improvement in anxiety and depression during remission periods has not been specifically addressed in the literature to date. Nevertheless, there are some possible explanations. First of all, the termination of the $\mathrm{CH}$ attack associated with remission may in itself directly improve the mood of $\mathrm{CH}$ patients, under the assumption that mood changes during the pre- and post-ictal phases of a $\mathrm{CH}$ attack contribute to the presence of anxiety and depression [32]. Next, nocturnal $\mathrm{CH}$ attacks are common during an active cluster bout, which can lead to sleep disturbance as well as depression [11, 21, 31]. In light of this, the lack of nocturnal $\mathrm{CH}$ attacks during the remission period may be another reason that improvement in mood is observed. Lastly, neuroimaging studies of $\mathrm{CH}$ have reported functional changes in multiple brain networks in relation to cluster bout status [28]. Given the shared anatomical location of the pain matrix and depression processing site, mood improvements may be a consequence of brain changes between active cluster bouts and remission periods.

The present study has several methodological limitations. First, despite the multicenter recruitment of study patients, our results were based on an analysis of data from a hospital-based dataset. In particular, the number of coexisting migraine in the $\mathrm{CH}$ patients was resultantly small and similar to the migraine prevalence of general population. Therefore, the findings in the current study require confirmation through additional population-based studies. Second, we estimated the risk of anxiety and depression in $\mathrm{CH}$ and coexisting migraine during active cluster bouts based on a cross-sectional analysis. Third, the median follow-up time interval used to assess for a change in anxiety and depression between active cluster bouts and remission was around 3 months. This may be considered short, given that the duration of the remission period varies from months to years. Longer observational studies are required to confirm the presence of dynamic changes in anxiety and depression across repeated phases of active cluster bouts and remission periods. Fourth, the multivariableadjusted logistic regression analyses to assess the association of $\mathrm{CH}$ with anxiety and depression did not adjust for unmeasured potential confounders, such as socioeconomic status, educational level, sleep disorders, more specific physical illness including low back pain, diabetes, and other headache disorders. Further studies complementing this weakness are required to better verify a specific association between $\mathrm{CH}$, coexisting migraine, anxiety, and depression. Fifth, we used the tests of GAD-7 and PHQ-9 to investigate the associations between $\mathrm{CH}$, anxiety, and depression. However, these tests do not confirm the diagnosis of anxiety and depression, but only represent their state, which should be kept in mind. Sixth, due to the moderate sample size of our study, there was a small number of the group of $\mathrm{CH}$ and coexisting migraine having anxiety and depression. This may raise a problem throughout the statistical process. In this regard, the results should be interpreted with caution. Finally, the $10.5 \%$ patients had probable $\mathrm{CH}$, which might influence the results.

\section{Conclusions}

We have quantified the risk of anxiety and depression in $\mathrm{CH}$ and coexisting migraine during active cluster bouts. We have shown that coexisting migraine is a significant influencer on psychiatric comorbidities in patients with $\mathrm{CH}$. Furthermore, anxiety and depression are dynamically altered between active cluster bout and remission periods, suggesting that psychiatric comorbidities may be cyclical in a similar manner to the cluster bouts observed in $\mathrm{CH}$. Further clinical and neuroimaging studies are required to elucidate on the possible mechanisms underlying our findings. 


\section{Supplementary information}

Supplementary information accompanies this paper at https://doi.org/10. 1186/s10194-020-01120-7.

Additional file 1: Table S1. Association of cluster headache and coexisting migraine with suicidal iedea of Patient Health Questionnaire-9.

\section{Abbreviations}

aOR: Multivariable-adjusted odds ratio; $\mathrm{CCH}$ : Chronic cluster headache; $\mathrm{CH}$ : Cluster headache; Cl: Confidence interval; ECH: Episodic cluster headaches; GAD-7: Generalized anxiety disorder 7-item scale; ICHD-3: The third edition of the international classification of headache disorders; IRB: Institutional review board; KCHR: Korean cluster headache registry; OR: Odds ratio; PHQ-9: Patient health questionnaire 9-item scale

\section{Acknowledgements}

None.

\section{Authors' contributions}

B.S.K., K.Y.P., and S.J.C. devised the idea and designed the study for this article. B.S.K., P.W.C., B.K.K., M.J.L., J.W.P., M.K.C., J.Y.A., D.W.B., T.J.S., J.H.S., K.O., D.K., J.M.K., S.K.K., Y.J.C., J.M.C., H.S.M., C.S.C., K.Y.P., and S.J.C. contributed the acquisition of the data and conducted the data analyses and data interpretations. B.S.K., K.Y.P., and S.J.C. drafted the initial manuscript. P.W.C., B.K.K., M.J.L., J.W.P., M.K.C., J.Y.A., D.W.B., T.J.S., J.H.S., K.O., D.K., J.M.K., S.K.K Y.J.C., J.M.C., H.S.M., and C.S.C. made the critical revision of the paper with important intellectual content. All authors reviewed and approved the final manuscript. All authors agreed to be responsible aspects of the work in ensuring that questions related to the accuracy or integrity of any part of the work are appropriately investigated and resolved.

\section{Funding}

No financial relationships relevant to this publication were disclosed.

\section{Availability of data and materials}

The data used in the present study are available from the corresponding author on reasonable request.

\section{Ethics approval and consent to participate}

The study protocol was approved by the ethics committee in each participating hospital and complied with the Declaration of Helsinki and Good Clinical Practice guidelines. All patients fully understood the study aims and gave informed written consent before their participation.

\section{Consent for publication}

not applicable.

\section{Competing interests}

Drs. BS Kim, PW Chung, BK Kim, MJ Lee, JW Park, JY Ahn, DW Bae, TJ Song, JH Sohn, K Oh, D Kim, JM Kim, SK Kim, YJ Choi, JM Chung, HS Moon, CS Chung, and KY Park report no conflict of interest.

Dr. Chu was involved as a site investigator for a multicenter trial sponsored by Otsuka Korea, Allergan, Ildong Pharmaceutical Co., LTD, Novartis International AG, and Eli Lilly and Company. He worked an advisory member for Teva, and received lecture honoraria from Allergan Korea, Handok-Teva and Yuyu Pharmaceutical Company in the past 24 months.

Dr. Cho was involved as a site investigator of multicenter trial sponsored Otsuka Korea, Allergan, Ildong Pharmaceutical Co., LTD, Novartis International AG, Eli Lilly and Company. Parexel Korea Co., Ltd., and and received lecture honoraria from Allergan Korea, WhanIn Pharm Co., LTD, Handok-Teva and Yuyu Pharmaceutical Company.

\section{Author details}

'Department of Neurology, Bundang Jesaeng General Hospital, Daejin Medical Center, Seongnam, South Korea. '2Department of Neurology, Kangbuk Samsung Hospital, Sungkyunkwan University School of Medicine, Seoul, South Korea. ${ }^{3}$ Department of Neurology, Eulji Hospital, Eulji University, Seoul, South Korea. ${ }^{4}$ Department of Neurology, Neuroscience Center, Samsung Medical Center, Sungkyunkwan University School of Medicine, Seoul, South Korea. ${ }^{5}$ Department of Neurology, Uijeongbu St.Mary's Hospital,
The Catholic University of Korea College of Medicine, Uijeongbu, South Korea. ${ }^{6}$ Department of Neurology, Severance Hospital, Yonsei University College of Medicine, Seoul, South Korea. ${ }^{7}$ Department of Neurology, Seoul Medical Center, Seoul, South Korea. ${ }^{8}$ Department of Neurology, College of Medicine, The Catholic University of Korea, Suwon, South Korea. ${ }^{9}$ Department of Neurology, Ewha Womans University Seoul Hospital, College of Medicine, Seoul, South Korea. ${ }^{10}$ Department of Neurology, Chuncheon Sacred Heart Hospital, Hallym University College of Medicine, Chuncheon, South Korea. ${ }^{11}$ Department of Neurology, Korea University College of Medicine, Seoul, South Korea. ${ }^{12}$ Department of Neurology, Chungnam National University College of Medicine, Daejeon, South Korea. ${ }^{13}$ Department of Neurology, Gyeongsang National University College of Medicine, Jinju, South Korea. ${ }^{14}$ Dr. Choi's Neurology Clinic, Jeonju, South Korea.

${ }^{15}$ Department of Neurology, Inje University College of Medicine, Seoul, South Korea. ${ }^{16}$ Department of Neurology, Chung-Ang University Hospital, 102 Heukseok-ro, Dongjak-gu, Seoul 06973, South Korea. ${ }^{17}$ Department of Neurology, Dongtan Sacred Heart Hospital, Hallym University College of Medicine, Keun Jae Bong-gil 7, Hwaseong, Gyeonggi-do 18450, South Korea.

Received: 18 January 2020 Accepted: 28 April 2020

Published online: 29 May 2020

\section{References}

1. May A, Schwedt TJ, Magis D, Pozo-Rosich P, Evers S, Wang SJ (2018) Cluster headache. Nat Rev Dis Primers 4:18006

2. Matharu MS, Goadsby PJ (2002) Trigeminal autonomic cephalgias. J Neurol Neurosurg Psychiatry 72(Suppl 2):ii19-ii26

3. Nesbitt AD, Goadsby PJ (2012) Cluster headache. Bmj. 344:e2407

4. Torelli P, Lambru G, Manzoni GC (2006) Psychiatric comorbidity and headache: clinical and therapeutical aspects. Neurol Sci 27(Suppl 2):S73-S76

5. Hamelsky SW, Lipton RB (2006) Psychiatric comorbidity of migraine. Headache. 46(9):1327-1333

6. Antonaci F, Nappi G, Galli F, Manzoni GC, Calabresi P, Costa A (2011) Migraine and psychiatric comorbidity: a review of clinical findings. J Headache Pain 12(2):115-125

7. Dresler T, Caratozzolo S, Guldolf K, Huhn Jl, Loiacono C, Niiberg-Pikksoot T et al (2019) Understanding the nature of psychiatric comorbidity in migraine: a systematic review focused on interactions and treatment implications. J Headache Pain 20(1):51

8. Minen MT (2016) Begasse De Dhaem O, kroon Van Diest a, powers S, Schwedt TJ, Lipton $\mathrm{R}$, et al. migraine and its psychiatric comorbidities. J Neurol Neurosurg Psychiatry 87(7):741-749

9. Robbins MS (2013) The psychiatric comorbidities of cluster headache. Curr Pain Headache Rep 17(2):313

10. Torkamani M, Ernst L, Cheung LS, Lambru G, Matharu M, Jahanshahi M (2015) The neuropsychology of cluster headache: cognition, mood, disability, and quality of life of patients with chronic and episodic cluster headache. Headache. 55(2):287-300

11. Louter MA, Wilbrink LA, Haan J, van Zwet EW, van Oosterhout WP, Zitman FG et al (2016) Cluster headache and depression. Neurology 87(18):1899_ 1906

12. Choong CK, Ford JH, Nyhuis AW, Joshi SG, Robinson RL, Aurora SK et al (2017) Clinical characteristics and treatment patterns among patients diagnosed with cluster headache in U.S. healthcare claims data. Headache 57(9):1359-1374

13. Jumani L, Kumari R, Kataria D, Kumar V, Usama SM, Chandnani A et al (2019) Depression among patients with chronic cluster headaches. Cureus 11(10): e5912

14. Pohl H, Gantenbein AR, Sandor PS, Schoenen J, Andrée C (2019) The impact of depressive symptoms on the burden of cluster headache: results of the EUROLIGHT cluster headache project, an internet-based, cross-sectional study of people with cluster headache. Cephalalgia Rep 2: 2515816319888211

15. Rausa M, Cevoli S, Giannini G, Favoni V, Contin SA, Zenesini C et al (2019) State and trait anger and its expression in cluster headache compared with migraine: a cross-sectional study. Neurol Sci 40(11):2365-2370

16. Pohl H, Gantenbein AR, Sandor PS, Schoenen J, Andree C (2020) Interictal burden of cluster headache: results of the EUROLIGHT cluster headache project, an internet-based, cross-sectional study of people with cluster headache. Headache 60(2):360-369 
17. Jurgens TP, Gaul C, Lindwurm A, Dresler T, Paelecke-Habermann Y, SchmidtWilcke T et al (2011) Impairment in episodic and chronic cluster headache. Cephalalgia 31(6):671-682

18. Robbins MS, Bronheim R, Lipton RB, Grosberg BM, Vollbracht S, Sheftell FD et al (2012) Depression and anxiety in episodic and chronic cluster headache: a pilot study. Headache. 52(4):600-611

19. D'Amico D, Centonze V, Grazzi L, Leone M, Ricchetti G, Bussone G (1997) Coexistence of migraine and cluster headache: report of 10 cases and possible pathogenetic implications. Headache. 37(1):21-25

20. Vollesen AL, Benemei S, Cortese F, Labastida-Ramirez A, Marchese F, Pellesi $L$ et al (2018) Migraine and cluster headache - the common link. J Headache Pain 19(1):89

21. Gaul C, Christmann N, Schroder D, Weber R, Shanib H, Diener HC et al (2012) Differences in clinical characteristics and frequency of accompanying migraine features in episodic and chronic cluster headache. Cephalalgia 32(7):571-577

22. Steinberg A, Fourier C, Ran C, Waldenlind E, Sjostrand C, Belin AC (2018) Cluster headache - clinical pattern and a new severity scale in a Swedish cohort. Cephalalgia 38(7):1286-1295

23. Headache Classification Committee of the International Headache Society (IHS) The International Classification of Headache Disorders, 3rd edition. Cephalalgia : an international journal of headache. 2018;38(1):1-211

24. Sohn JH, Choi YJ, Kim BK, Chung PW, Lee MJ, Chu MK et al (2018) Clinical features of probable cluster headache: a prospective, cross-sectional multicenter study. Front Neurol 9:908

25. Cho SJ, Lee MJ, Kim BK, Moon HS, Chung PW, Sohn JH et al (2019) Clinical features of chronic cluster headache based on the third edition of the international classification of headache disorders: a prospective multicentre study. PLoS One 14(8):e0221155

26. Chung PW, Lee MJ, Park JW, Sohn JH, Kim BK, Chu MK et al (2019) Differences of cluster headache on the basis of sex in the Korean cluster headache registry. Headache 59(10):1722-1730

27. Liang JF, Chen YT, Fuh JL, Li SY, Liu CJ, Chen TJ et al (2013) Cluster headache is associated with an increased risk of depression: a nationwide population-based cohort study. Cephalalgia 33(3):182-189

28. Yang FC, Chou KH, Kuo CY, Lin YY, Lin CP, Wang SJ (2018) The pathophysiology of episodic cluster headache: insights from recent neuroimaging research. Cephalalgia 38(5):970-983

29. Ferraro S, Nigri A, Bruzzone MG, Demichelis G, Pinardi C, Brivio L et al (2019) Cluster headache: insights from resting-state functional magnetic resonance imaging. Neurol Sci 40(Suppl 1):45-47

30. Buture A, Boland JW, Dikomitis L, Ahmed F (2019) Update on the pathophysiology of cluster headache: imaging and neuropeptide studies. Pain Res 12:269-281

31. Barloese MC (2015) Neurobiology and sleep disorders in cluster headache. J Headache Pain 16:562

32. Snoer A, Lund N, Beske R, Hagedorn A, Jensen RH, Barloese M (2018) Cluster headache beyond the pain phase: a prospective study of 500 attacks. Neurology 91(9):e822-ee31

\section{Publisher's Note}

Springer Nature remains neutral with regard to jurisdictional claims in published maps and institutional affiliations.

Ready to submit your research? Choose BMC and benefit from:

- fast, convenient online submission

- thorough peer review by experienced researchers in your field

- rapid publication on acceptance

- support for research data, including large and complex data types

- gold Open Access which fosters wider collaboration and increased citations

- maximum visibility for your research: over $100 \mathrm{M}$ website views per year

At $\mathrm{BMC}$, research is always in progress.

Learn more biomedcentral.com/submissions 